Methods and Results The role of neutrophilic inflammation and PAR-1 was investigated in two models of murine pneumococcal pneumonia (serotype 2 (D39) and serotype 19F (EF3030)) by using the most clinically advanced PAR-1 antagonist, SCH530348. Neutrophil depletion and chemokine neutralisation studies were also performed. Samples were analysed by immunohistochemistry, cytology, flow cytometry, ELISA and microbiological techniques. Our models were characterised by evidence of intra-alveolar coagulation, increased neutrophil recruitment to areas of bacterial infection and increased PAR-1 expression (demonstrated by quantitative image-analysis). Neutrophil depletion protected mice against barrier disruption but resulted in compromised host defence. In contrast, PAR-1 antagonist treatment significantly reduced neutrophil recruitment to the bronchoalveolar space without being detrimental to host defence. Markers of alveolar leak, coagulation activation and pro-inflammatory cytokines and chemokines (IL-1 $\beta$, CXCL1, CCL2 and CCL7) were also attenuated. Neutralisation studies demonstrated that IL-1 $\beta$ and CCL7, but not CXCL1 and CCL2, played a key role in neutrophil recruitment to the airspaces in this model. Translational studies were performed to examine by flow cytometry the CXC and CC chemokine receptor expression on neutrophils from blood and BALF of mechanically ventilated CAPinduced ARDS patients and controls. CXCR1 and CXCR2 expression on BALF neutrophils was higher in CAP-ARDS patients compared to controls. Additionally, chemokine expression patterns on neutrophils from CAP-ARDS patients changed within different compartments, evidenced by decreased expression of CXCR1 and increased expression of CXCR2, CCR1, CCR2 and CCR3 on neutrophils from BALF compared with blood.

Conclusion These data provide preclinical proof-of-concept that recently developed PAR-1 antagonists may offer a novel therapeutic approach for controlling or preventing alveolar barrier dysfunction and excessive neutrophilic inflammation in pneumococcal pneumonia without compromising host defence. Furthermore, they highlight a role for chemokine receptor switching in CAP-ARDS with important implications for future targeting of these chemokine receptors.

\section{S101 SRC/BCR-ABL INHIBITION WITH DASATINIB IN STERILE AND NON-STERILE ACUTE LUNG INFLAMMATION}

${ }^{1} \mathrm{JG}$ Macfarlane, ${ }^{2} \mathrm{DA}$ Dorward, ${ }^{2} \mathrm{CD}$ Lucas, ${ }^{1} \mathrm{JA}$ Scott, ${ }^{1} \mathrm{MH}$ Ruchaud-Sparagano, ${ }^{3} \mathrm{CMA}$ Khan, ${ }^{2} \mathrm{AG}$ Rossi, ${ }^{1} \mathrm{AJ}$ Simpson. ${ }^{1}$ Institute of Cellular Medicine, Newcastle Upon Tyne, UK; ${ }^{2}$ MRC Centre for Inflammation Research, Edinburgh, UK; ${ }^{3}$ Institute of Cell and Molecular Biosciences, Newcastle Upon Tyne, UK

10.1136/thoraxjnl-2014-206260.107

Introduction and objectives Adult respiratory distress syndrome (ARDS) is a commonly fatal complication of lung infection and inflammation, with no effective treatment. It is characterised by excessive neutrophil influx and degranulation into the lungs, with alveolar leak and severe hypoxia. Src family tyrosine kinases are critical in integrin-dependent neutrophil degranulation. Dasatinib is a $\mathrm{Src} / \mathrm{Bcr}$-abl inhibitor used in chronic myeloid leukaemia. We investigated our hypothesis that extracellular neutrophil degranulation could be inhibited by dasatinib in vitro and would modulate the inflammatory response in vivo in models of infective and sterile lung injury.

Methods Whole blood and isolated blood neutrophils from healthy volunteers were pre-treated with dasatinib and treated with neutrophil stimuli or live bacteria. Degranulation was measured by granule receptor expression and presence of extracellular granule products. Other neutrophil functions were assessed, including adhesion, L-selectin shedding, chemotaxis, phagocytosis, oxidative burst, bacterial killing and apoptosis. Neutrophilic lung inflammation was induced in mice using intratracheal E. coli or hydrochloric acid.

Results In vitro, dasatinib inhibited neutrophil degranulation in response to lipopolysaccharide derived from E. Coli 026:B6, fMLF and Staphylococcus aureus at concentrations above 100 $\mathrm{nM}$, with no effect on neutrophil viability or apoptosis. Integrindependent functions including adhesion, chemotaxis and phagocytosis in adherent conditions were impaired, but phagocytosis was unaffected in whole blood. Intracellular oxidative burst was maintained, with normal bacterial killing, but extracellular superoxide anion release was impaired.

In vivo, dasatinib had modest effects on the pro-inflammatory response to E. coli, reducing\%interstitial neutrophils, alveolar myeloperoxidase and $\mathrm{TNF} \alpha$ at $1 \mathrm{mg} / \mathrm{kg}$ and alveolar lactoferrin at $10 \mathrm{mg} / \mathrm{kg}$. Bacterial killing was impaired in a dose dependent fashion, with associated alveolar leak and systemic toxicity at 10 $\mathrm{mg} / \mathrm{kg}$. In sterile acid injury, $5 \mathrm{mg} / \mathrm{kg}$ dasatinib reduced\%neutrophils, degranulation (interstitial CD11b/alveolar lactoferrin) and monocyte-chemotactic protein-1 (MCP-1) in the alveolar space, but induced detrimental effects at $10 \mathrm{mg} / \mathrm{kg}$.

Conclusions The pan-Src kinase inhibitor dasatinib modifies multiple pro-inflammatory neutrophil functions in vitro and in vivo with an impairment in bacterial killing observed in infective lung injury. In the context of sterile inflammation, manipulation of neutrophil degranulation also alters the inflammatory environment and this approach warrants further study as a therapeutic strategy in ARDS.

\section{S102 LIPOXIN A4 IMPROVES EFFEROCYTOSIS VIA INHIBITION OF THE HMGB1 IN HUMAN ALVEOLAR MACROPHAGES}

${ }^{1} \mathrm{Q}$ Wang, ${ }^{1} \mathrm{D}$ Parekh, ${ }^{1} \mathrm{VK}$ D'Souza, ${ }^{1} \mathrm{R}$ Dancer, ${ }^{1} \mathrm{JM}$ Patel, 'D Bartis, ${ }^{1} \mathrm{~F}$ Gao, ${ }^{2} \mathrm{Q}$ Lian, ${ }^{2} \mathrm{~S}$ Jin, ${ }^{1}$ DR Thickett. ' Centre for Translational Inflammation and Respiratory Research, School of Clinical and Experimental Medicine, University of Birmingham, Birmingham, UK; ${ }^{2}$ Department of Anesthesia and Critical Care, Second Affiliated Hospital of Wenzhou Medical University, Zhejiang, China

\subsection{6/thoraxjnl-2014-206260.108}

Introduction Effective clearance of apoptotic cells by macrophages, termed efferocytosis, is a pre-requisite for successful resolution of inflammation. High mobility group box protein 1 (HMGB1), is an alarmin that may promote inflammation as well as suppress phagocytosis. Lipoxin $\mathrm{A}_{4}$, represents one of a unique class of lipid mediators that possess a wide spectrum of antiinflammatory and pro-resolution actions. We hypothesised that lipoxin $\mathrm{A}_{4}$ may promote both apoptosis in neutrophils, and stimulate macrophage efferocytosis, acting as an antagonist to HMGB-1.

Methods Neutrophils were obtained from healthy volunteers and cultured for $24 \mathrm{~h}$ with or without lipoxin $\mathrm{A}_{4}$. Apoptosis of neutrophils was determined with Annexin V/SyTox staining by flow cytometry. HMGB-1 levels in Acute Respiratory Distress Syndrome (ARDS) bronchoalveolar lavage fluid (BALF) was measured by ELISA. The effects of HMGB-1 and lipoxin $\mathrm{A}_{4}$ upon alveolar macrophage efferocytosis was assessed by measuring the ingestion of CMFDA labelled apoptotic neutrophils by flow cytometry. The PI3K (P85) protein expression was measured by western blotting. 
Results Treatment of lipoxin A4 $(100 \mathrm{nM})$ increased the apoptosis of neutrophils $(\mathrm{p}=0.0244)$, and reduced the dead $(\mathrm{p}=0.0238)$ and necrotic neutrophils $(\mathrm{p}=0.0358)$ compared to control $(\mathrm{n}=8)$. BALF from patients with ARDS suppressed efferocytosis of apoptotic neutrophils. The effects of BALF correlated with HMGB-1 levels in the BALF fluid. HMGB-1 decreased efferocytosis $(p<0.05)$ in a dose dependent manner, and reached a significant effect at $150 \mathrm{ng} / \mathrm{ml}(\mathrm{p}=0.008)$. Lipoxin A4 increased the efferocytosis $(p<0.05)$ of alveolar macrophages in a dose dependent manner, and reached the maximal effect at $100 \mathrm{nM}(\mathrm{p}=0.008)$. Moreover, lipoxin A4 (100 nM) blocked the decreased efferocytosis response to HMGB-1 $(150 \mathrm{ng} / \mathrm{ml}) \quad(\mathrm{p}=0.005, \mathrm{n}=8)$. The lipoxin A4 beneficial effects were abrogated by ALX antagonist, BOC-2 ( $<<0.05)$ and PI3K inhibitor $(\mathrm{p}<0.05)$.

Conclusions Lipxin $\mathrm{A}_{4}$ in vitro promotes the apoptosis but not necrosis of neutrophils. In tandem it stimulates efferocytosis of alveolar macrophages. Elevated HMGB-1 in ARDS BALF suppresses efferocytosis. Lipoxin $\mathrm{A}_{4}$ can block these effects of HMGB-1. The effect of lipoxin A4 increasing efferocytosis was through ALX-PI3K signalling pathways. Lipoxin $\mathrm{A}_{4}$ may therefore have potential as a therapeutic agent to promote the resolution of neutrophilic inflammation in ARDS.

\section{COPD outcomes}

\section{S103 NO LOSS IN EFFICACY FOLLOWING SWITCH FROM SALMETEROL/FLUTICASONE COMBINATION TO INDACATEROL MONOTHERAPY IN PATIENTS WITH MODERATE COPD: THE INSTEAD STUDY}

${ }^{1}$ Andrea Rossi, ${ }^{2}$ Thys van der Molen, ${ }^{3}$ Ricardo Del Olmo, ${ }^{4}$ Alberto Papi, ${ }^{5}$ Luis Webhe, ${ }^{6}$ Matthew Quinn, ${ }^{6}$ Chengxing Lu, ${ }^{7}$ David Young, ${ }^{7}$ Ray Cameron, ${ }^{8}$ Enrica Bucchioni, ${ }^{6}$ Pablo Altman. 'Pulmonary Unit, Azienda Ospedaliera Universitaria Integrata, Verona, Italy; ${ }^{2}$ University Medical Center Groningen, University of Groningen, Groningen, The Netherlands; ${ }^{3}$ CIDEA Foundation, Buenos Aires, Argentina; ${ }^{4}$ University of Ferrara, Ferrara, Italy; ${ }^{5}$ Instituto Ave Pulmo, Buenos Aires, Argentina; ${ }^{6}$ Novartis Pharmaceuticals Corporation, NJ, USA; ${ }^{7}$ Novartis Horsham Research Centre, Horsham, UK; ${ }^{8}$ Novartis Farma, Saronno, Italy

\subsection{6/thoraxjnl-2014-206260.109}

Introduction Many patients with low risk of COPD exacerbations receive twice-daily (bid) LABA/ICS, salmeterol/fluticasone (SFC), for maintenance treatment. This study evaluated the effect of switching these patients to a once-daily (od) LABA, indacaterol, monotherapy.

Methods INSTEAD was a 26-week double-blind, double-dummy study in patients aged $\geq 40$ years, with moderate COPD (postbronchodilator $\mathrm{FEV}_{1} \quad 50-80 \%$ predicted) and no exacerbations in the past 12 months, who were receiving SFC 50/500 $\mu \mathrm{g}$ bid for $\geq 3$ months prior to study entry. Patients were randomised (1:1) to continue with SFC 50/500 $\mu$ g or to be switched (with no washout) to indacaterol $150 \mu \mathrm{g}$. The primary objective was to demonstrate non-inferiority of indacaterol to SFC, measured by trough $\mathrm{FEV}_{1}$ after 12 weeks (non-inferiority margin: $60 \mathrm{~mL}$ ). Trough FEV1 was also evaluated at 4, 8 and 26 weeks. TDI and SGRQ-C total scores were evaluated at Weeks 12 and 26; the annualised rate of exacerbations and safety were evaluated over 26 weeks.

Results A total of581 patients were randomised (indacaterol: 293; SFC: 288); 85.4\% completed the study. The primary endpoint was met, with a LSM difference in trough $\mathrm{FEV}_{1}$ between indacaterol and SFC of $-9 \mathrm{~mL}(95 \% \mathrm{CI}:-45$ to $26 \mathrm{~mL}$; $\mathrm{p}=0.002$ for $\mathrm{NI})$. There were no significant differences between treatments in trough $\mathrm{FEV}_{1}$ at any of the other visits (Baseline and Weeks 4, 8 and 26). The TDI and SGRQ-C total scores and their responder rates were similar between two treatments, at both Weeks 12 and 26 (Table 1). During the 26 week treatment period, $79.5 \%$ and $74.7 \%$ of patients in the indacaterol and SFC groups, respectively, experienced no exacerbations. There was no statistically significant difference between treatments in the rate of all COPD exacerbations per year, with rates of 0.57 vs 0.67 , respectively (RR 0.86 [95\% CI 0.62 , 1.20]; $\mathrm{p}=0.367$ ). Adverse events (AEs) and serious AEs were comparable between the treatment arms.

Conclusion Indacaterol was non-inferior to SFC in terms of bronchodilation and showed similar efficacy in terms of breathlessness, health status, and exacerbation rate indicating that this group of patients can be switched from SFC to indacaterol 150 $\mu \mathrm{g}$ with no loss in efficacy.

\section{S104 DOUBLE-BLIND MULTI-CENTRE RANDOMISED CONTROLLED TRIAL OF VITAMIN D3 SUPPLEMENTATION IN COPD (VIDICO)}

${ }^{1} \mathrm{AR}$ Martineau, ${ }^{1} \mathrm{~W}$ James, ${ }^{1} \mathrm{RL}$ Hooper, ${ }^{1} \mathrm{NC}$ Barnes, ${ }^{1} \mathrm{DA}$ Jolliffe, ${ }^{2} \mathrm{~A}$ Bhowmik, ${ }^{1}$ RK Rajakulasingam, ${ }^{3} \mathrm{AB}$ Choudhury, ${ }^{4} \mathrm{DE}$ Simcock, ${ }^{5} \mathrm{CJ}$ Corrigan, ${ }^{5} \mathrm{CM}$ Hawrylowicz, ${ }^{1} \mathrm{CJ}$ Griffiths. 'Queen Mary University of London, London, UK; ${ }^{2}$ Homerton Hospital, London, UK; ${ }^{3}$ Queen's Hospital, Romford, UK; ${ }^{4}$ Royal London Hospital, London, UK; ${ }^{5}$ King's College London, London, UK

\subsection{6/thoraxjnl-2014-206260.110}

Introduction and objectives Inadequate vitamin D status is common in patients with COPD, and it associates with susceptibility to upper respiratory infection (URI) - a major precipitant of exacerbation. Multi-centre trials of vitamin D supplementation for prevention of exacerbation and URI in COPD are lacking. We therefore conducted a multi-centre double-blind randomised placebo-controlled trial of vitamin D supplementation for the prevention of moderate/severe exacerbation and URI in adults with COPD.

Methods Two hundred and forty patients were allocated to receive a 2-monthly oral dose of $3 \mathrm{mg}$ vitamin $\mathrm{D}_{3}$ or placebo for one year. Co-primary outcomes were time to first moderate/

Abstract S103 Table 1 TDI and SGRQ scores

\begin{tabular}{|c|c|c|c|c|c|c|}
\hline & \multicolumn{2}{|l|}{ Week 12} & \multirow[b]{2}{*}{ Treatment difference $(95 \% \mathrm{Cl})$} & \multicolumn{2}{|l|}{ Week 26} & \multirow[b]{2}{*}{ Treatment difference $(95 \% \mathrm{Cl})$} \\
\hline & Indacaterol & SFC & & Indacaterol & SFC & \\
\hline TDI total score & $1.89(0.499)^{\dagger}$ & $1.69(0.509)^{\dagger}$ & $0.20(-0.32,0.72)$ & $2.58(0.543)^{\dagger}$ & $2.70(0.552)^{\dagger}$ & $-0.12(-0.71,0.48)$ \\
\hline SGRQ-C total score & $32.8(1.58)^{\dagger}$ & $32.9(1.61)^{\dagger}$ & $-0.10(-1.9,1.8)$ & $33.1(1.87)^{\dagger}$ & $33.5(1.93)^{\dagger}$ & $-0.40(-2.5,1.6)$ \\
\hline TDI responder ( $\geq 1$ unit), $\%$ & 61.4 & 61.0 & $0.97(0.65,1.46)^{\#}$ & 68.7 & 69.4 & $0.88(0.58,1.35)^{\#}$ \\
\hline SGRQ-C responder ( $\geq 4$ unit),\% & 44.7 & 42.4 & $1.12(0.76,1.63)^{\#}$ & 49.6 & 48.8 & $1.03(0.70,1.52)^{\#}$ \\
\hline
\end{tabular}

${ }^{\dagger}$ Data are LSM (SE): \#odds ratio; LSM, least square means; TDI, transition dyspnoea index; SGRQ-C, St George Respiratory Questionnaire for COPD 